\title{
Cooperation, Trust and Games in Wireless Networks
}

\author{
John S. Baras and Tao Jiang \\ Institute for Systems Research and \\ Electrical and Computer Engineering Department \\ University of Maryland \\ College Park, MD 20742 \\ baras@isr.umd.edu, tjiang@glue.umd.edu
}

\subsection{Introduction}

As an important concept in network security, trust is interpreted as a set of relations among agents participating in the network activities. Trust relations are based on the previous behavior of an agent within a protocol. Trust establishment in distributed and resource-constraint networks, such as mobile ad hoc networks (MANETs), sensor networks and ubiquitous computing systems, is much more difficult but more crucial than in traditional hierarchical architectures, such as the Internet and base station- or access point-centered wireless LANs. Generally, this type of distributed networks have neither pre-established infrastructures, nor centralized control servers or trusted third parties (TTP). The trust information or evidence used to evaluate trustworthiness is provided by peers, i.e. the agents that form the network. Furthermore, resources (power, bandwidth, computation etc.) are normally limited because of the wireless and ad hoc environment, so the trust evaluation procedure should only rely on local information. Schemes that depend only on local interaction also have the desired emergent property that enables fast reaction to network member changes, topology changes and security changes that frequently happen in mobile networks. Therefore, the essential and unique properties of trust management in this new paradigm of wireless networking, as opposed to traditional centralized approaches are: uncertainty and incompleteness of trust evidence, trust value is between -1 and 1 ; locality in trust information exchange; distributed computation.

Trust establishment is a process starting from a small set of agents who are known to be trustworthy. For example, the first few peers to join a network are often known to be trustworthy, while the majority are neutral, i.e. with trust value 0 . They are subsequently evaluated by agents who have direct interaction with them. Those evaluating agents are either the physical or logical neighbors of target agents. Based on their observations and evidence, they are able to provide opinions on the target agent, to build the trust value (also called reputation) of the target agent. The whole network therefore evolves as the local interactions iterate from "isolated trust islands" to "a 
connected trust graph." Our interest is to discover rules and policies that establish trust-connected networks using only local interactions, to understand the impact of local interactions on the whole network and also to find the conditions under which trust spreads to a maximum set, as well as the parameters that speed up or slow down this transition.

There have been several works on trust computation based on interactions with one-hop physical neighbors. In [2], for instance, first-hand observations are exchanged between neighboring nodes, where node $A$ adjusts his opinion for $B$, based on how close $B$ 's evidence is to $A$ 's previous opinion about another node $C$. It provides an innovative model to link nodes' trustworthiness with the quality of the evidence they provide. Our work emphasizes the inference of trust value instead of generating the direct trust, which is similar to [7] and [8], where weighted averages were used to aggregate multiple votes for trust evaluation and provided promising results on using this simple local interaction rule to correctly evaluate trust in distributed networks. Particularly in [7], different kinds of malicious behaviors have been simulated and their results showed that by ranking nodes according to the trust value, the network application (in their case, file downloading in $\mathrm{p} 2 \mathrm{p}$ networks) doesn't get affected by malicious nodes. However, the results in both [7] and [8] are based on simulation. In this chapter, we analyze a local interaction rule using graph theory and provide a theoretical justification for network management that facilitates trust propagation.

In wireless networks such as mobile ad hoc networks and sensor networks, most of the functions (routing, mobility management, and security) must rely on cooperation between nodes. In addition, such cooperation utilizes local information and local (between neighbors) interactions. This is probably the most important difference between this type of networks and traditional networks, such as the Internet and cellular networks.

In the wireless networks of interest in this chapter, nodes are not under the control of any central authority. In other words, each node is its own authority. The network is generated in a more distributed and asynchronous manner. In this situation, the most reasonable assumption is that each node will try to maximize its benefit by exploiting the network, even if this means adopting a selfish behavior. This selfishness means that nodes are not willing to participate, without additional incentives, in the common networking functions, such as route discovery, packet forwarding and security management, which are always resources consuming, including power batteries and bandwidth consumption.

Over the last few years, there has been an increasing amount of research on designing mechanisms to encourage nodes to collaborate. Basically, the approaches taken can be divided into two categories: one is based on incentive techniques, which normally rely on various kinds of trust or reputation systems to promote cooperation and circumvent misbehaving nodes $[2,3,9]$; the other is inspired from game theory, where payoffs are assigned to different strategies of nodes, and Nash equilibria in non-cooperative games are considered to be the optimal and stable solutions $[5,13]$.

In our chapter, the interactions among nodes are also modeled as games, which are cooperative games rather than non-cooperative games, where players always 
conflict. In cooperative games, players form coalitions to obtain the optimum payoffs. The key assumption that distinguishes cooperative game theory from noncooperative game theory is that players can negotiate effectively [10]. We will discuss how negotiation can help to form the grand coalition that includes all players together. Another way to form a grand coalition is through a trust establishment mechanism: nodes which do not cooperate will be penalized by the trust establishment mechanism. How trust establishment mechanisms can help in cooperative games is also analyzed. Furthermore, we show that trust establishment and evolution of cooperation go hand in hand by viewing the whole network as a distributed dynamical system.

As discussed, trust computation is distributed and restricted to only local interactions in a MANET. Each node, as an autonomous agent, makes the decision on trust evaluation individually. The decision is based on information it has obtained by itself or from its neighbors. Those aspects are analogous to situations in statistical mechanics of complex systems with game theoretic interactions. Game theory, and more specifically the theory of evolutionary games, provide the framework for modeling individual interactions. This circle of ideas has a lot in common with randomized optimization methods from statistical physics.

One of the simplest local interaction models is the Ising model [11], which describes the interaction of magnetic moments or spins, where some spins seek to align with one another (ferromagnetism), while others try to anti-align (antiferromagnetism). The Ising spin model consists of $n$ spins. Each spin is either in position "up" or "down." Any configuration of spins is denoted as $\mathbf{s}=\left\{s_{1}, s_{2}, \ldots, s_{n}\right\}$, where $s_{i}=1$ or -1 indicating spin $i$ is up or down respectively. A Hamiltonian, or energy, for a configuration $\mathbf{s}$ is given by

$$
H(\mathbf{s})=-\frac{1}{T} \sum_{\forall i \in V, j \in N_{i}} J_{i j} s_{i} s_{j}-\frac{m H}{T} \sum_{i} s_{i}
$$

where $T$ is the temperature. The first term represents the interaction between spins. The second term represents the effect of the external (applied) magnetic field. In the Ising model the local interaction "strengths" are all equal to a parameter $J$. In the more complex case of spin glass the $J_{i j}$ are different and may even come from random processes [11].

The problem of computing the ground state (global minimum of energy) for the Ising model (and even more so for spin glasses) is an NP-hard problem. There are $2^{n}$ possible configurations for the model, the computation becomes infeasible when $n$ gets large. So we must use heuristic methods to find low energy configurations. As proposed in [1], we could imagine that the spins try to reduce their own frustration (or energy) individually, and come up with an interesting cooperative game. In game theoretic terms, the payoff for node $i$, when the graph has a configuration $\mathbf{s}=\left\{s_{1}, s_{2}, \ldots, s_{n}\right\}$, is

$$
\pi_{i}=\sum_{j \in N_{i}} J_{i j} s_{i} s_{j}
$$


When $J_{i j}=1$, the agents are rewarded for aligning their spin states; when $J_{i j}=-1$ they want to take on opposite states (anti-align their spins) in order to maximize their payoffs. Agents interact in order to maximize their own payoffs.

This model provides the inspiration for our approach, as it can be directly used for distributed trust computation. Let $s_{i}$ be the trust value assigned to node $i$, where $s_{i} \in\{-1,1\}$. Node $i$ will be assigned a trust value according to the opinion of the majority of its neighbors. We set $J_{i j}=1, \forall j \in N_{i}$. Then the payoff of agent $i$ is $\pi_{i}=s_{i} \sum_{j \in N_{i}} s_{j}$. In order to maximize $\pi_{i}, i$ will set $s_{i}$ with the same sign as $\sum_{j \in N_{i}} s_{j}$, which is actually the same value as neighbor majority vote. Simulations using Simulated Annealing (SA) show that the average payoff of the whole network is a function of the temperature $T$ in the Ising model. High temperatures, in the trust computation context, mean that the agents are very conservative and not willing to change their trust values, the payoffs are near 0 , which is the expected payoff for a random set of $s_{i}$ from $\{-1,1\}$. While, as the temperature decreases (aggressive agents), the algorithm becomes greedier and payoffs increase, most of the nodes will reach agreement. Recently there has been very strong interest in the application and extension of such optimization schemes from the statistical mechanics of spin glasses and associated games to optimization and other problems in information technology [11].

In the Ising model, and the more complex models of spin glasses, an important characteristic is phase transition phenomena. It is observed that when the temperature is high, all the spins behave nearly independently (no long-range correlation), whereas when temperature is below a critical temperature $c_{0}$, all the spins tend to stay the same (i.e., cooperative behavior). Phase transitions are also studied in evolutionary prisoner's dilemma games [14]. Phase transition is a common phenomenon that takes place in any combinatorial structure, where a large combinatorial structure can be modeled as a system consisting of many locally interacting components. A phase transition corresponds to a change in some global (macroscopic) parameter of the system as the local parameters are varied. Distributed trust computation is essentially a cooperative game where nodes interact with their neighbors locally.

The structure of the chapter is as follows. In Section 11.2 we develop the network model and the framework of cooperative games for analyzing cooperation among the agents. In Section 11.3 we analyze the cooperative game framework and show that agent cooperation can be achieved employing negotiations between the agents. We also develop a dynamic distributed trust mechanism framework and demonstrate that it can also induce cooperation among agents, albeit without negotiations. In Section 11.4 we investigate the dynamic evolution of both cooperative games and trust mechanisms and establish certain quantitative measures and characteristics of the "spread" of cooperative behavior among agents. Finally, Section 11.5 contains our conclusions and a brief description of future research directions. 


\subsection{Problem formulation}

\subsubsection{System model}

The network is modeled as an undirected graph $G(V, E)$. Throughout this chapter, we use the terms node, player and agent interchangeably, where a node $i$ is an element in the set $V$. Nodes are players that play games among themselves. Since we only consider direct interaction among nodes, nodes only play games with their neighbors, which are denoted as:

$$
\mathcal{N}_{i} \triangleq\{j \mid(i, j) \in E\} \subseteq\{1, \ldots, N\} \backslash\{i\} .
$$

The neighbor set of agent $i, \mathcal{N}_{i}$, can represent the set of agents with which $i$ is allowed to communicate (giving rise to a logical interconnection network), or the set of agents which $i$ can sense, transit or receive information from (physical wireless communication links).

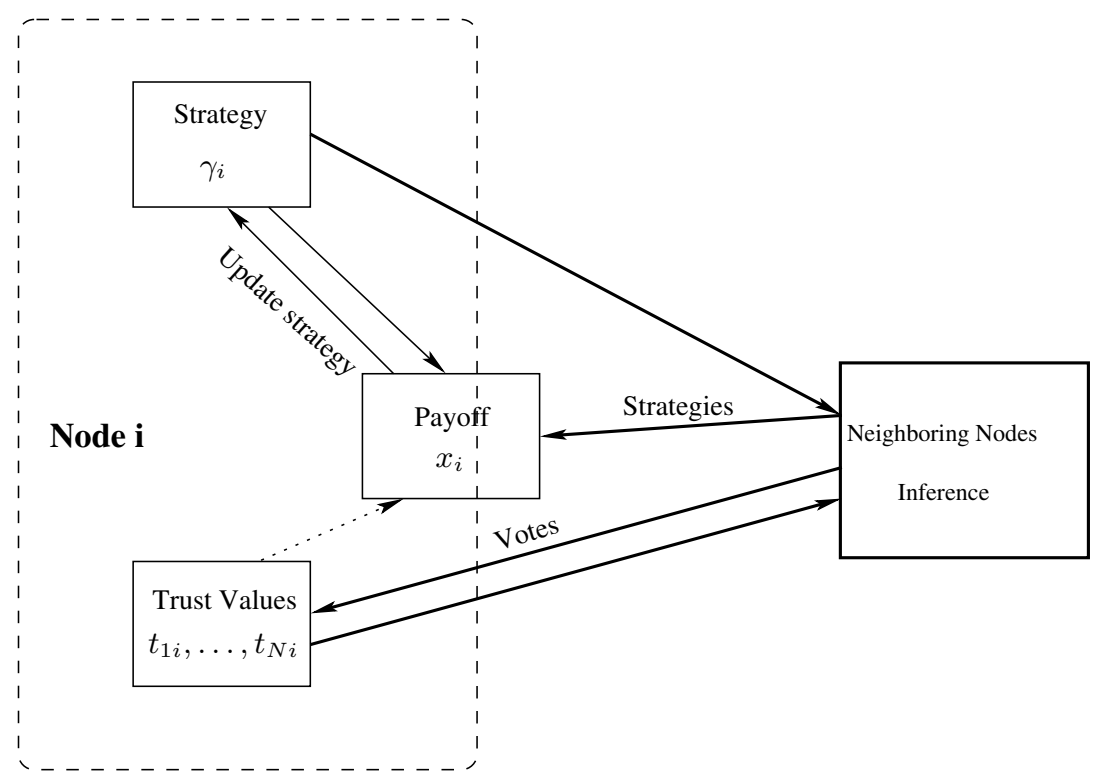

Fig. 11.1. System operation block-graph for a typical node.

In our model, each node has a self-defined playing strategy, which is denoted by $\gamma_{i}$ for node $i$. Another characteristic of each node is its trust values, which are dependent on the opinions of other nodes. Trust values of a node can be different for different players. For instance, $t_{j i}$ and $t_{k i}$ are the trust values of $i$ provided by distinct player $j$ and $k$, and possibly $t_{j i} \neq t_{k i}$. Fig. 11.1 is a block graph demonstrating how nodes interact among their neighbors, where the payoff of node $i$ after playing games is represented as $x_{i}$. The procedure is summarized as the following three rules: 
- Strategy updating rule: as shown in Fig. 11.1, nodes update strategies based on their own payoffs. They tend to choose rules that obtain the maximum payoffs.

- Payoff function: the payoffs are functions of the strategies of all participants. For a specific node, the payoff only depends on strategies of its neighbors and itself.

- Trust computation rule: trust values are computed based on votes, which are provided by neighbors and are related to the history (strategies and trust values) of the target node. Since trust values eventually have impact on the payoff of the node, there is a dotted line in Fig. 11.1 from trust values to payoff to represent their implicit relation.

For simplicity, we assume the system is memoryless. All values are dependent only on parameter values at most one time step in the past. Therefore, the system can be modeled as a discrete-time system:

$$
\begin{aligned}
\gamma_{i}(t+1) & =f^{i}\left(x_{i}(t), \gamma_{i}(t), \gamma_{j}(t), t_{i j}(t)\right) \\
t_{i k}(t) & =g^{i}\left(t_{i j}(t), v_{j k}(t)\right) \quad \forall k \in N \\
x_{i}(t) & =h^{i}\left(\gamma_{i}(t), \gamma_{j}(t)\right) \\
v_{i j}(t) & =p^{i}\left(\gamma_{j}(t), t_{j i}(t)\right)
\end{aligned}
$$

where $j$ stands for all neighbors of $i$, and $v_{i j}$ is the value node $i$ votes for $j$. In Section 11.4, we will analyze the dynamics of the system, especially the effect of trust propagation on the formation of cooperation. We first introduce the basic element of this system: the cooperative games among neighboring nodes.

\subsubsection{Games}

In this part, we give the formal definitions of the interaction games. In our work, we consider two-person games with perfect information, say, player (or node) $P_{1}$ interacts with player (or node) $P_{2}$.

Definition 1 (Strategy). A strategy $\gamma_{i}$ for $P_{i}$ is the alternative $P_{i}$ chooses based on the information it currently holds. The set of all strategies of $P_{i}$ is called his strategy set (space), and it is denoted by $\Gamma_{i}$.

Definition 2 (Payoff). The payoff of player $P_{i}$ is the function of the strategies of both players, which is denoted by $x_{i}=f_{i}\left(\gamma_{1}, \gamma_{2}\right)$.

In a game, two rational players choose their strategies based on the information they have, and aim to achieve the optimum payoff. Games are generally divided into two categories: non-cooperative games and cooperative games. The essential difference of these two types of games is that in cooperative games players are allowed to negotiate while in non-cooperative games players play the game for their own sake. Therefore, in cooperative games, correlated mixed strategies are allowed, and the payoff can be transferred from one player to the other (though not always linearly). In what follows we will compare two different games by providing simple example games; our game model is based on a simple cooperative game and the interactions among neighbors. 


\section{Non-cooperative vs. cooperative games}

One of the most well-known models in two-player non-cooperative games is the prisoner's dilemma. In the prisoner's dilemma, the strategy sets of both players are $\Gamma_{i}=$ \{cooperate, defect $\}$. Then there are four combinations for $\left(\gamma_{1}, \gamma_{2}\right)$ and the payoffs of two players are assigned in a matrix form as shown in Table 11.1, where

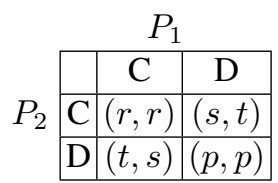

Table 11.1. Payoff matrix of prisoner's dilemma.

"C" stands for cooperate and "D" for defect. The payoffs are related to whether players cooperate or not and to what extent. For each possible pair of strategies, $r$ is the "reward" payoff that each player receives if both cooperate, $p$ is the "punishment" that each receives if both defect, $t$ is the "temptation" that each receives if he alone defects and $s$ is the "sucker's" payoff that he receives if he alone cooperates. The payoffs satisfy the following chain of inequalities:

$$
t>r>p>s \text {. }
$$

Players try to maximize their payoffs. For player $P_{1}$, strategy $\mathrm{D}$ is strictly dominant to the strategy $\mathrm{C}$ : whatever his opponent does, he is better off choosing $\mathrm{D}$ than $\mathrm{C}$. By symmetry, D also strictly dominates $\mathrm{C}$ for player $P_{2}$. Thus two "rational" players will defect and receive a payoff of $p$, while two "irrational" players can cooperate and receive greater payoff $r$.

In cooperative games, players are allowed to negotiate and use the strategies according to their committed agreement. Under such an assumption, rational players either cooperate at the same time or defect simultaneously. If two players do not cooperate, the payoff they get is called the disagreement vector $\mathbf{f}^{*} \in \mathbf{R}^{2}$. If they cooperate, the players negotiate about which point in the set of feasible payoffs $L \in$ $\mathbf{R}^{2}$ they will agree upon. So in cooperative games we need to investigate: 1 ) whether players are willing to reach a consensus on which feasible payoff to realize; 2) how to allocate the payoffs among the players. We can analyze a simple cooperative game that is a modification of the prisoner's dilemma: the disagreement vector $\mathbf{f}^{*}=(p, p)$, for simplicity let $p=0$ and let the payoffs be defined as

$$
\begin{aligned}
x_{1} & =f\left(a_{2}\right)-c a_{1} \\
x_{2} & =f\left(a_{1}\right)-c a_{2} \\
a_{1}+a_{2} & \leq E
\end{aligned}
$$

where $a_{1}$ and $a_{2}$ are some limited resources (with limit $E$ ) shared by two players, such as money or bandwidth in the network context, and $f$ be a concave function. Fig. 11.2 depicts an example of the players' payoffs. 


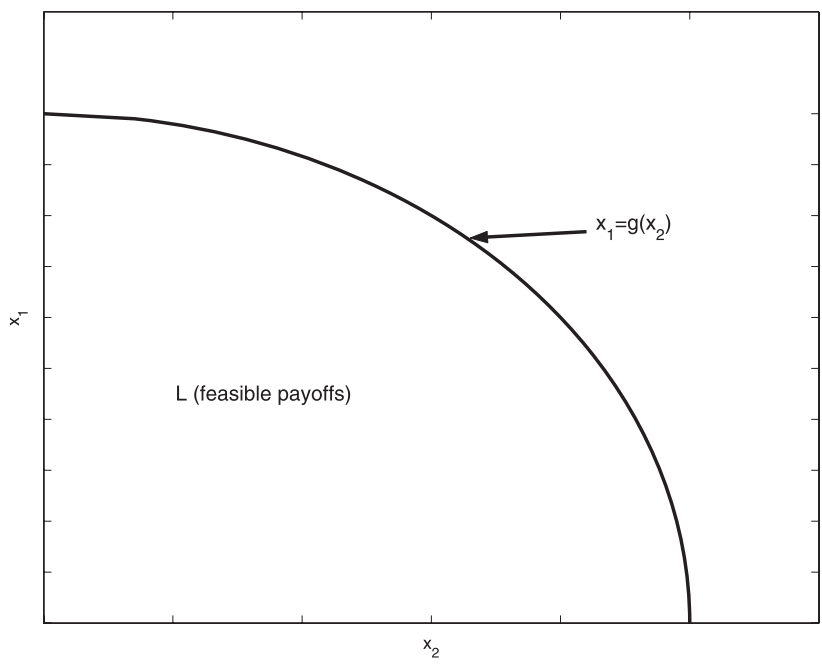

Fig. 11.2. Illustration of a two-player cooperative game.

The negotiation result $\mathbf{x}=\left(x_{1}, x_{2}\right)$ satisfies the following conditions

1. $\mathrm{x} \in L$ (feasibility);

2. $\mathbf{x} \geq \mathbf{f}^{*}$ (rationality);

3. $\mathrm{x}^{\prime} \in L, \mathrm{x}^{\prime} \geq \mathrm{x}$ imply $\mathrm{x}^{\prime}=\mathrm{x}$ (Pareto-optimality).

Then the boundary of the compact, convex feasible set $D=L \cap\left\{\mathbf{x}: \mathbf{x} \geq \mathbf{f}^{*}\right\}$, i.e. the curve $x_{1}=g\left(x_{2}\right)$ in Fig. 11.2, is the set of candidates for negotiation. Then the question is: on which point the agents would agree on if they cooperate? This will be discussed in Sect. 11.3.

\section{Games on networks}

In this chapter, we consider cooperative games on networks, where nodes play cooperative games with their neighbors iteratively. Assume that at each time step, two neighboring nodes only play the game once. Cooperative games are normally represented by the characteristic function form which is a finite set $N=\{1, \ldots, N\}$, the set of players and a function (characteristic function) $v: 2^{N} \rightarrow \mathbf{R}$ defined on all subsets (coalitions) of $N$ with $v(\emptyset)=0$. We denote such a game as $\Gamma=(N, v)$. Define $S$, a subset of $N$, as a coalition if all nodes in $S$ cooperate. Then $v(S)$ is interpreted as the maximum utility (payoff) $S$ can get without the cooperation of the rest of the players $N \backslash S$. In order to simplify our analysis, we assume the payoff only depends on the interacting two parties and the feasible payoff set of the two-player game is shown in Fig. 11.2. Suppose $y_{i j}$ is the payoff of $i$ from the game between $i$ and $j$. Since games are played on networks, $y_{i j} \neq 0$ only if $i$ and $j$ are neighbors, 
and set $y_{i j}=0$ if $i=j$ or $i$ and $j$ are not neighbors. For instance, consider two neighboring nodes $i$ and $j$ and let $S=\{i, j\}$, then

$$
v(S)=\max \left\{y_{i j}+y_{j i}\right\} .
$$

Apparently, the payoffs that maximize $v(s)$ are on the Pareto frontier of the convex set $L$. Substitute $y_{i j}=g\left(y_{j i}\right)$ into (11.7), and we can derive the payoffs that maximize $v(s)$, denoted as $\left(x_{i j}, x_{j i}\right)$. In a geometric interpretation, $\left(x_{i j}, x_{j i}\right)$ is the point on the boundary of $L$ from where a tangent to $L$ can be drawn with slope -1 . It is obvious that $\mathbf{x}=\left(x_{i j}, x_{j i}\right)$ satisfies the negotiation conditions.

The following are assumptions made and used in this chapter:

- The games are with transferable utility, i.e., payoffs were given in linearly transferable utility.

- The cooperation is bilateral, i.e. for two neighboring nodes, either both cooperate or none cooperates. This is because there is no incentive for a node to altruistically contribute without receiving some payoff.

- Nodes cooperate with all the neighbors in the same coalition. If $i$ is in coalition $S, j \in \mathcal{N}_{i}$ and $j \in S$, then $i$ cooperates with $j$.

As we defined, a coalition is a subset of nodes that cooperate with all their neighbors in the coalition. Among all coalitions, there are so-called maximum coalitions which are not subsets of any other coalition, i.e., if $S$ is a maximum coalition, then $\forall i \in S, j \notin S, i$ and $j$ do not cooperate with each other. In this chapter, all coalitions are maximum coalitions, so we omit maximum from now on. We could easily find the characteristic function of our cooperative game, which is the summation of the payoffs from all cooperative pairs in the coalition, as:

$$
v(S)=\sum_{i, j \in S} x_{i j} .
$$

Notice that $\forall i, v(\{i\})=0$. We denote the cooperative game defined from (11.8) as $\Gamma=(N, v)$.

In the next section, we will describe the details of the system model. Based on the model, we will investigate stable solutions for enforcing cooperation among nodes, and demonstrate two efficient methods for achieving such cooperation: negotiation and trust mechanism.

\subsection{Cooperation in games}

\subsubsection{Cooperative games with negotiation}

In Section 11.2.2, we reviewed and defined games, especially cooperative games that are used in our interaction model. In this section, we investigate the impact of the games on the collaboration in a network. First we start with a simple fact.

Lemma 1. If $\forall i, j, x_{i j}+x_{j i} \geq 0$, then $\Gamma=(N, v)$ is a superadditive game. 
Proof. Suppose $S$ and $T$ are two disjoint sets $(S \cap T=\emptyset)$, then

$$
\begin{aligned}
v(S \cup T) & =\sum_{i, j \in S \cup T} x_{i j}=\sum_{i, j \in S} x_{i j}+\sum_{i, j \in T} x_{i j}+\sum_{i \in S, j \in T}\left(x_{i j}+x_{j i}\right) \\
& =v(S)+v(T)+\sum_{i \in S, j \in T}\left(x_{i j}+x_{j i}\right) \geq v(S)+v(T) .
\end{aligned}
$$

The last inequality holds by our assumption that $x_{i j}+x_{j i} \geq 0$.

The main concern in cooperative games is how the total payoff from a partial or complete cooperation of the players is divided among the players. A payoff allocation is a vector $\mathbf{x}=\left(x_{i}\right)_{i \in N}$ in $\mathbf{R}^{N}$, where each component $x_{i}$ is interpreted as the payoff allocated to player $i$. We say that an allocation $\mathrm{x}$ is feasible for a coalition $S$ iff $\sum_{i \in S} x_{i} \leq v(S)$.

When we think of a reasonable and stable payoff, the first thing that comes to mind is a payoff that would give each coalition at least as much as the coalition could enforce itself without the support of the rest of the players. In this case, players couldn't get better payoffs if they form separate coalitions different from the grand coalition $N$. The set of all these payoff allocations of the game $\Gamma=(N, v)$ is called the core and is formally defined as the set of all $n$-vectors $\mathbf{x}$ satisfying the linear inequality:

$$
\begin{array}{r}
\mathbf{x}(S) \geq v(S) \quad \forall S \subset N, \\
\mathbf{x}(N)=v(N),
\end{array}
$$

where $\mathrm{x}(S)=\sum_{i \in S} x_{i}$ for all $S \subset N$. If $\Gamma$ is a game, we will denote its core by $C(\Gamma)$. It is known that the core is possibly empty. Therefore, it is necessary to discuss existence of the core for the game $\Gamma$. We first give the definition of a family of common games: convex games [6]. The convexity of a game can be defined in terms of the marginal contribution of each player, which plays the role of first difference of the characteristic function $v$. Convexity of $v$ can be defined in terms of the monotonicity of its first differences. The first difference (or the marginal contribution of player $i$ ) $d_{i}: 2^{N} \rightarrow \mathbf{R}$ of $v$ with respect to player $i$ is

$$
d_{i}(S)= \begin{cases}v(S \cup\{i\})-v(S) & \text { if } i \notin S \\ v(S)-v(S \backslash\{i\}) & \text { if } i \in S .\end{cases}
$$

A game is said to be convex, if for each $i \in N, d_{i}(S) \leq d_{i}(T)$ holds for any coalition $S \subset T$.

Lemma 2. $\Gamma(N, v)$ is a convex game.

Proof. For $\Gamma, d_{i}(S)=\sum_{j \in S, j \neq i}\left(x_{j i}+x_{i j}\right)$. Taking two sets $S \subset T$,

$$
d_{i}(T)-d_{i}(S)=\sum_{j \in T \cap S^{c}}\left(x_{j i}+x_{i j}\right) \geq 0 .
$$


The core of a convex game is nonempty ([6]), thus $C(\Gamma) \neq \emptyset$. By Lemma 2, we have the following theorem,

Theorem 1. $\Gamma=(N, v)$ has a nonempty core.

Now let's find one of the payoff allocations that are in the core. For any pair of players $(i, j)$, suppose the payoff allocation of the game between $i$ and $j$ is $\left(\hat{x}_{i j}, \hat{x}_{j i}\right)$. Then we have the following

Corollary 1. If the payoff allocation satisfies $\hat{x}_{i j} \geq 0$ and $\hat{x}_{j i} \geq 0$, then the payoff allocation $\hat{x}_{i}=\sum_{j \in \mathcal{N}_{i}} \hat{x}_{i j}$ is in the core $C(\Gamma)$.

Proof. Take an arbitrary subset $S \subset N$,

$$
\hat{x}(S)=\sum_{i \in S} \hat{x}_{i}=\sum_{i, j \in S} \hat{x}_{i j}+\sum_{i \in S, j \notin S} \hat{x}_{i j} \geq \sum_{i, j \in S} \hat{x}_{i j}=v(S) ;
$$

the inequality holds because $\hat{x}_{i j} \geq 0, \forall i, j \in N$.

Because we only consider transferable utility games, $\hat{x}_{i j}+\hat{x}_{j i}=x_{i j}+x_{j i} \geq 0$. Therefore $\left(\hat{x}_{i j}, \hat{x}_{j i}\right)$ could be constructed in the following way:

$$
\hat{x}_{i j}= \begin{cases}x_{i j} & \text { if } x_{i j} \geq 0, x_{j i} \geq 0 \\ x_{i j}+\lambda_{i j} x_{j i} & \text { if } x_{i j}<0, x_{j i}>0 \\ \left(1-\lambda_{i j}\right) x_{j i} & \text { if } x_{i j}>0, x_{j i}<0\end{cases}
$$

where $0 \leq \lambda_{i j}=\lambda_{j i} \leq 1$, and $\hat{x}_{i j} \geq 0$ is achieved by carefully choosing $\lambda_{i j}$.

Obviously, the payoff allocation we provided in Corollary 1 is a set of points in the core, while there generally exist more points in the core that are not covered in the Corollary. However, this solution indicates a way to encourage cooperation in the whole network. The players that have positive gain can negotiate with their neighbors by sacrificing certain gain (offering their partial gain $\lambda x_{j i}$ ). Though they cannot achieve their best possible payoff, they can set up a cooperative relation with their neighbors. This is definitely beneficial for the players who negotiate and sacrifice, since without cooperation they cannot get anything. This solution is also efficient and scalable, because players only need to negotiate with their direct neighbors.

Thus we established cooperative games among nodes in the network, and described an efficient way to achieve cooperation throughout the network. In the next section, we are going to discuss solutions by employing trust mechanisms, which do not require negotiation and the assumption on $x_{i j}+x_{j i} \geq 0$ can also be relaxed.

\subsubsection{Trust mechanism}

Trust is a useful incentive for encouraging nodes to collaborate. Nodes who refrain from cooperation get lower trust value and will be eventually penalized because other nodes tend to only cooperate with highly trusted ones. From Fig. 11.1 and the corresponding system equations, the trust values of each node will eventually influence its payoff. Let's assume, for node $i$, that the loss of not cooperating with node $j$ 
is a nondecreasing function of $x_{j i}$, because the more $j$ loses, the more effort $j$ undertakes to reduce the trust value of $i$. Denote the loss for $i$ being non-cooperative with $j$ as $l_{i j}=f\left(x_{j i}\right)$ and $f(0)=0$. For simplicity, assume the characteristic function is a linear combination of the original payoff and the loss, which is shown as

$$
v^{\prime}(S)=\sum_{i, j \in S} x_{i j}-\sum_{i \in S, j \notin S} f\left(x_{j i}\right) .
$$

The game with characteristic function $v^{\prime}$ is denoted as $\Gamma^{\prime}\left(N, v^{\prime}\right)$. We then have

Theorem 2. If $\forall i, j, x_{i j}+f\left(x_{j i}\right) \geq 0$, then $C\left(\Gamma^{\prime}\right) \neq \emptyset$ and $x_{i}=\sum_{j \in N} x_{i j}$ is a point in $C\left(\Gamma^{\prime}\right)$.

Proof. First we prove $\Gamma^{\prime}$ is a convex game, given $x_{i j}+f\left(x_{j i}\right) \geq 0$. We have that $\forall i \in N$ in $\Gamma^{\prime}$,

$$
d_{i}(S)=\sum_{j \in S, j \neq i}\left(x_{i j}+x_{j i}\right)-\sum_{k \notin S} f\left(x_{k i}\right)+\sum_{j \in S, j \neq i} f\left(x_{i j}\right) .
$$

Letting $S \subset T$,

$$
\begin{aligned}
d_{i}(T)-d_{i}(S) & =\sum_{j \in T \cap S^{c}}\left(x_{i j}+x_{j i}\right)+\sum_{k \in T \cap S^{c}} f\left(x_{k i}\right)+\sum_{j \in T \cap S^{c}} f\left(x_{i j}\right) \\
& =\sum_{j \in T \cap S^{c}}\left(\left(x_{i j}+f\left(x_{j i}\right)\right)+\left(x_{j i}+f\left(x_{i j}\right)\right)\right) \geq 0 .
\end{aligned}
$$

Therefore $C\left(\Gamma^{\prime}\right)$ is nonempty. Next, we verify that $x_{i}=\sum_{j \in \mathcal{N}_{i}} x_{i j}$ is in the core. For any $S \in N$,

$$
\begin{aligned}
\sum_{i \in S} x_{i}-v(S) & =\sum_{i \in S} \sum_{j \in N} x_{i j}-\left(\sum_{i, j \in S} x_{i j}-\sum_{i \in S, k \notin S} f\left(x_{k i}\right)\right) \\
& =\sum_{i \in S, j \notin S}\left(x_{i j}+f\left(x_{j i}\right)\right) \geq 0 .
\end{aligned}
$$

Apparently, the payoff $x_{i}=\sum_{j \in \mathcal{N}_{i}} x_{i j}$ does not need any payoff negotiation. Thus we showed that by introducing a trust mechanism, all nodes are induced to collaborate with their neighbors without any negotiation.

In this section, we introduced two approaches that encourage all nodes in the network to cooperate with each other: 1) negotiation among neighbors; 2) trust mechanism. We proved that both approaches lead to a nonempty core for the cooperative game played in the network. However, we have only considered these two approaches separately, and the results are based on static settings. The more interesting problems are how these two intertwine and how the dynamics between the two approaches converge to a cooperative network - these are discussed in the next section. 


\subsection{Dynamics of cooperation}

We have analyzed the effect of a trust mechanism on the formation of cooperation. However, what we concentrated on in Section 11.3.2 is the final impact of trust on the payoffs at the steady state. In this section, we are going to discuss two dynamic behaviors in the system: trust propagation and game evolution.

\subsubsection{Trust propagation}

Trust propagation is concerned with how trust evidences (usually negative evidences) propagate from the victims (those who do not receive desired services from their neighbors) and how the trust evidences of a certain node reach its neighborhood and trigger off revocation. The consequence of revocation is that the neighbors refuse to cooperate with the poorly-trusted node and finally isolate it.

Our model is motivated by considering a group of agents each of whom must decide between two alternative actions (trust or distrust a certain node), and whose decisions depend explicitly on the actions of other members of the group. Apparently, the other members are those who are interacting with the agent. In economic terms, this entire class of problems is known generically as binary decisions with externalities. Though it appears as a very simple binary decision problem, it is relevant to surprisingly complex problems, such as statistical mechanics. The decision rule in our model is basically a threshold rule. Agents are usually reluctant to switch their decisions, because decisions usually require more resources and time. But once their individual threshold has been reached, even a single evidence can trigger them into switching from one state to another. Our decision rule, which is particularly simple, while capturing the essential features outlined above, is the following. Every node keeps a state that represents its opinion on a particular node, say node $i$ : 0 stands for distrust and 1 stands for trust. Suppose initially all nodes have the state 1, i.e., nodes first trust all others, but the state immediately changes if the node observes non-cooperation of the particular node $i$. We model the system evolving in discrete time. At each time step, a node observes the current states (either 0 or 1 ) of other nodes it interacts with, which we call its neighbors. The node adopts state 0 if at least a threshold fraction $\phi$ of its $k$ neighbors are in state 0 , else it adopts state 1 .

Because of the differences in knowledge, preferences and observational capabilities across the nodes, the threshold $\phi$ is allowed to be heterogeneous. $\phi$ is determined by the individual node, and can be modeled as drawn at random from a pre-defined distribution with pdf $\mathrm{f}(\phi)$. As we have discussed, to model the dynamics of the revocation, the states of all nodes are initially set to 1 . At a certain time, the noncooperative behavior of node $i$ is observed, then a fraction (usually very small, because the network is sparse) of the nodes are switched to state 0 . The whole network evolves at successive time steps, with all nodes updating their states in asynchronous order according to the threshold decision rule above. Once a node has switched to state 0 , it remains at 0 for the rest of the dynamics.

The main objective of trust propagation is to explore how the trust revocation depends on the network interactions. Because building relationships and exchanging 
information are both costly, especially for wireless ad hoc networks, the interactions tend to be very sparse, so we consider only the properties of networks with low (node) degree. Our approach concentrates on two quantities: (i) the probability that the revocation is accepted by a sufficiently large portion of the network (or a finite fraction for an infinite network) triggered from a single node (or small fraction of nodes) — we call these phenomena global revocation; and (ii) the expected size of the global revocation.

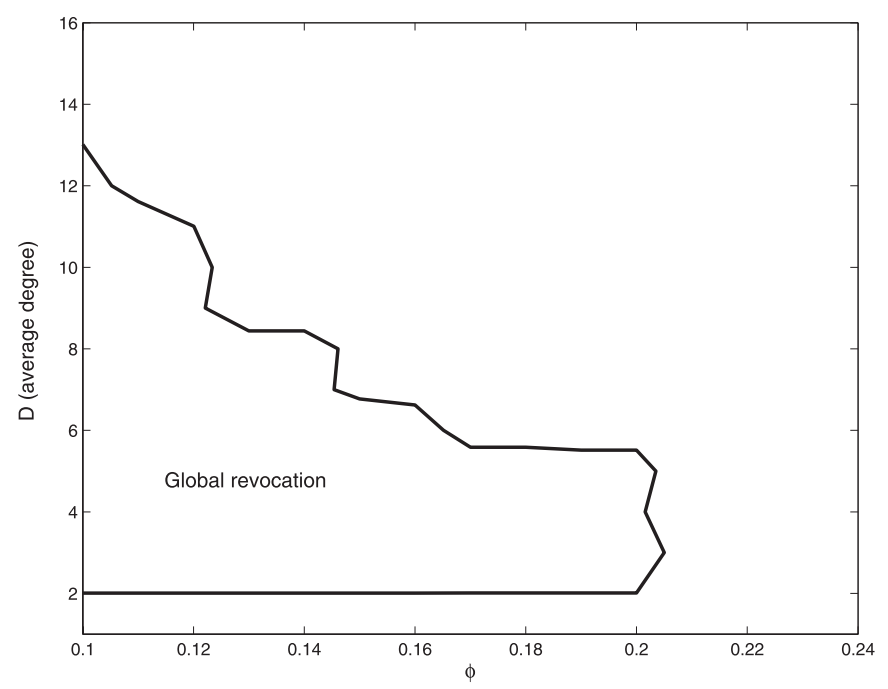

Fig. 11.3. Revocation windows for the threshold model. The network is a random graph based on Erdös and Rényi [4].

Fig. 11.3 graphically shows the condition for global revocation. For simplicity, we assume homogeneity, i.e., the threshold $\phi$ is the same for every node. The average (node) degree of the network [12] is given by $d$. The line encloses the region of the $(\phi, d)$ plane in which a large fraction $(80 \%)$ of the network nodes accept the revocation. Fig. 11.4 illustrates that the fraction of nodes accepting revocation changes with the threshold $\phi$, with fixed average (node) degree.

The phase transitions in Fig. 11.4 define the boundaries of the revocation windows. The exact solutions for the phase transitions are discussed in [15], which also provides the comparison of different network topologies. Therefore, the network topology and threshold value are crucial parameters for global revocation. This gives an important indication and reference for network management and decision control in sparse networks, where agents interact and make decisions based on information provided by their neighbors, and in collaboration with their neighbors. 


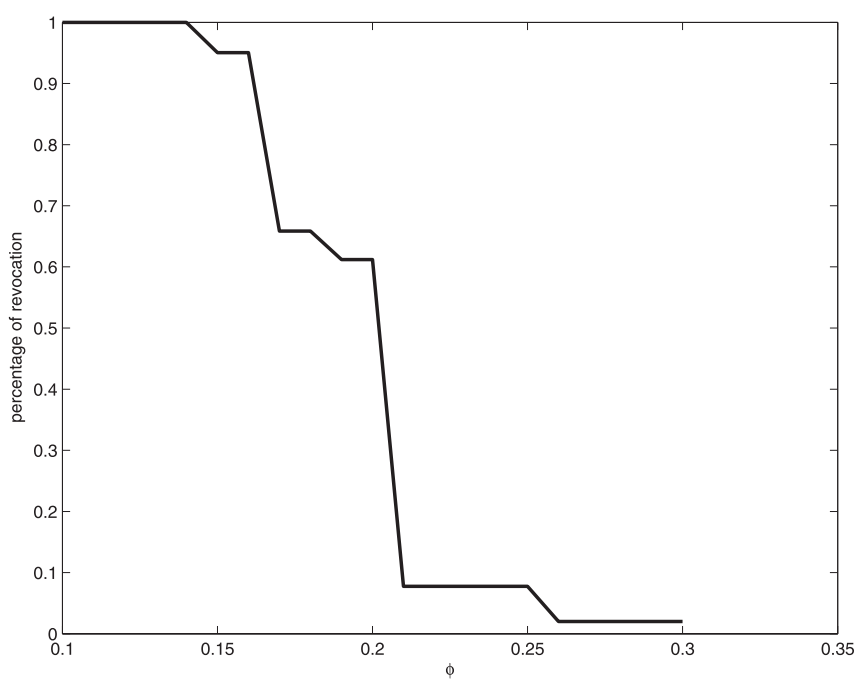

Fig. 11.4. Percentage of nodes accepting revocation vs. threshold $\phi, d=6$.

\subsubsection{Game evolution}

As shown in Sect. 11.3.2, the trust mechanism drives selfish nodes to sacrifice part of their benefits and thus promotes cooperation. In this section, the procedure and dynamics of such cooperation evolution are studied.

In this section, we assume that nodes either cooperate or do not cooperate with neighbors. $\gamma_{i j}=1$ denotes that node $i$ cooperates with its neighbor $j$, and $\gamma_{i j}=0$ denotes that it does not cooperate with $j$. We assume that the payoff when one of them does not cooperate is fixed as $(0,0)$, and as $\left(x_{i j}, x_{j i}\right)$ when both cooperate. If $x_{i j}<0$, we call the link $(i, j)$ a negative link for node $i$, and when the opposite holds a positive link. Since all nodes are selfish, nodes tend to cooperate with neighbors that are on positive links, while they do not wish to cooperate with neighbors on negative links. Meanwhile, the trust mechanism is employed, which aims to function as the incentive for cooperation. In this part, we assume that revocation and nullification of revocation can propagate throughout the network as discussed in Section 11.4.1.

In our evolution algorithm, each node maintains a record of its past experience by using the variable $\Delta_{i}(t)$. First define $x_{a, i}(t)$ as the payoff $i$ gains at time $t$ and $x_{e, i}(t)$ as the expected payoff $i$ can get at time $t$ if $i$ always chooses cooperation with all neighbors. Notice that the expected payoff can be different each time, since it depends on whether the neighbors cooperate or not at the specific time. Then compute the cumulative difference,

$$
\Delta_{i}(t)=\Delta_{i}(t-1)+\left(x_{a, i}(t)-x_{e, i}(t)\right),
$$

of the total payoff in the past minus the expected payoff if the node always cooperates. Each node chooses its strategy on the negative links by the following rule: 
- $\quad$ if $\Delta_{i}(t)<0$, node $i$ chooses to cooperate, i.e., $\gamma_{i j}=1, \forall j \in \mathcal{N}_{i}$.

- $\quad$ if $\Delta_{i}(t) \geq 0, \gamma_{i j}=0$, if $j \in \mathcal{N}_{i}$ and $x_{i j}<1$.

Notice that at time $0, \Delta_{i}(0)=0$. That is to say initially all nodes choose not to cooperate on the negative links, since they are inherently selfish. There are two other conditions that force non-cooperation strategies:

- nodes do not cooperate with neighbors that have been revoked.

- nodes do not cooperate with non-cooperative neighbors.

To summarize, as long as one of those aforementioned conditions is satisfied, nodes choose not to cooperate.

Since we allow and encourage nodes to rectify, i.e., to change their strategies from non-cooperation to cooperation, we define a temporal threshold $\tau$ in the trust propagation rule. Instead of always keeping 0 once the state is switched to 0 , as in Section 11.4.1, we allow the nullification of revocation (switch back to state 1) under the condition that the revocation has been nullified for $\tau$ consecutive time steps. $\tau$ also represents the penalty for being non-cooperative. $\tau$ needs to be large so that the non-cooperative nodes would rather switch to cooperate than get penalized. However, large $\tau$ will also reduce the payoff.

The detailed algorithm is shown in Fig. 11.5.

Suppose the total payoff of node $i$, if every node cooperates, is $x_{i}=\sum_{j \in \mathcal{N}_{i}} x_{i j}$. We have the following

Theorem 3. $\forall i \in N$ and $x_{i}>0$, there exists $\tau_{0}$, such that for a fixed $\tau>\tau_{0}$ :

1. The iterated game converges to Nash equilibrium.

2. $\Delta_{i}(t) / t \rightarrow 0$ as $t \rightarrow \infty$.

3. $i$ cooperates with all its neighbors for t large enough.

Proof. Nodes without negative links, will always cooperate, thus $\Delta_{i} \equiv 0$. Therefore, we only consider nodes with negative links. First we prove that for $t$ large enough $\Delta_{i}(t)<0$. Define for node $i$, the absolute sum of positive payoffs and negative payoffs as $x_{i}^{(p)}$ and $x_{i}^{(n)}$ respectively. Then

$$
x_{i}=x_{i}^{(p)}-x_{i}^{(n)} .
$$

Therefore the first payoff for node $i$ is $x_{a, i}(1)=x_{i}^{(p)}>0$ and $\Delta_{i}=x_{i}^{(n)}$. Define $T_{\max }$ as the maximum propagation delay in the network. Then at $t=T_{\max }$ all $i$ 's neighbors revoke $i$ because at time $t=1, i$ didn't cooperate, and the payoff now is $x_{a, i}\left(T_{\max }\right)=0$ and $\Delta_{i}\left(T_{\max }\right)=\Delta_{i}\left(T_{\max }-1\right)-x_{i} . i$ continues to get 0 payoff till all neighboring nodes have used the penalty interval $\tau$. It's easy to show that as $\tau$ is set large enough, $i$ eventually gets negative $\Delta_{i}$.

If $i$ follows the strategy rules in Fig. 11.5, $i$ starts to cooperate with all neighbors. The difference of the actual payoff and expected payoff is 0 from then on. Therefore $\Delta_{i}(t) / t \rightarrow 0$ as $t \rightarrow \infty$.

Assume node $i$ deviates to non-cooperation, then it will get negative cumulative payoff difference as discussed above. So node $i$ has no intention to deviate from 
Consider node $i$, and the initial settings are as follows:

- $\quad$ all the trust states are set to $s_{i j}=1, \forall j \in N$;

- the variable $\Delta_{i}(0)=0$.

Node $i$ chooses strategies and updates variables in each time step for $t=$ $1,2, \ldots$ :

1. The strategy on the game with neighbor $j$ is set according to the following rule:

- for negative links $\left(x_{i j}<0\right)$, choose non-cooperation strategy $\left(\gamma_{i j}=0\right)$ if $\Delta_{i}(t-1) \geq 0$;

- $\quad$ if $s_{i j}=0, \gamma_{i j}=0$;

- for all neighbors, $\gamma_{i j}=0$ iff $\gamma_{j i}=0$ (cooperation is bilateral);

- otherwise $\gamma_{i j}=1$.

2. For all $j \in \mathcal{N}_{i}$, update the trust state $s_{i j}$ if one of the following three conditions is satisfied, otherwise keep the previous state

- if $i$ accepts a revocation on node $j, s_{i j}=0$;

- if the revocation has been nullified for more than $\tau$ consecutive steps, set $s_{i j}=1$;

- $\quad$ if $\gamma_{j i}=0$, set $s_{i j}=0$;

3. Compute the actual payoff $x_{a, i}(t)$ and expected payoff $x_{e, i}(t)$, then get the cumulative difference $\Delta_{i}(t)$ by Eqn.( 11.12).

Fig. 11.5. Algorithm for game evolution modeling trust revocation.

cooperation. Therefore the game converges to its Nash equilibrium with all nodes cooperating.

We have also performed simulation experiments with our evolution algorithm. In the simulations, we didn't assume the condition that $\forall i, x_{i}>0$, instead the percentage of negative links is the simulation parameter. We can report that without this condition, our iterated game with the trust scheme can still achieve very good performance. Fig. 11.6 shows that cooperation is highly promoted under the trust mechanism. In Fig. 11.7, the average payoffs between the algorithm with strategy update and without strategy update are compared, which explains the reason why nodes converge to cooperation.

\subsection{Conclusions and Future Directions}

In this chapter we investigated fundamental methods by which collaboration in infrastructure-less wireless networks with mobile nodes can be induced, analyzed and evaluated. In this chapter we have also described a new framework within which the problem of distributed trust establishment and maintenance in a mobile ad hoc network (MANET) can be formulated and analyzed. 


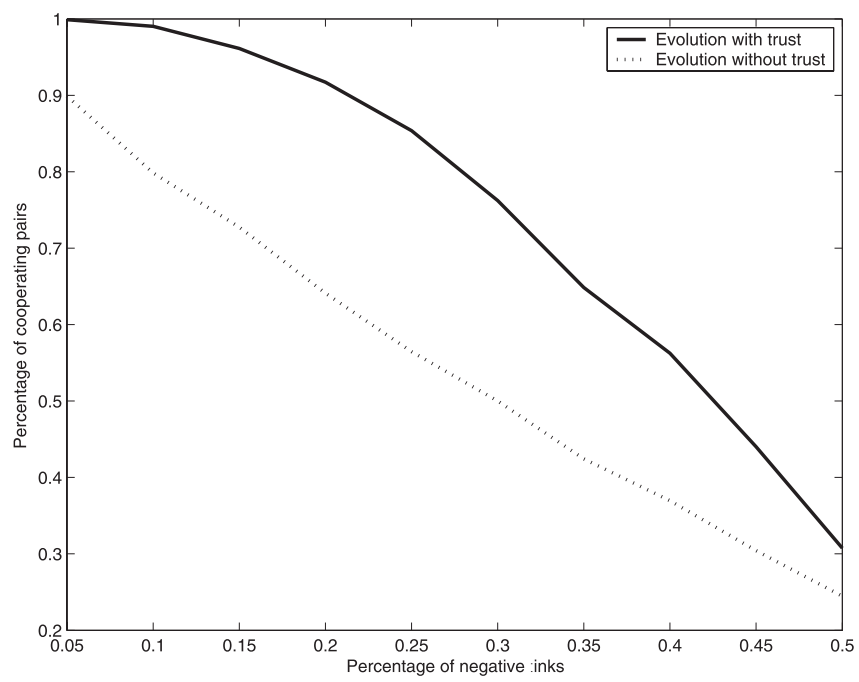

Fig. 11.6. Percentage of cooperating pairs vs. negative links.

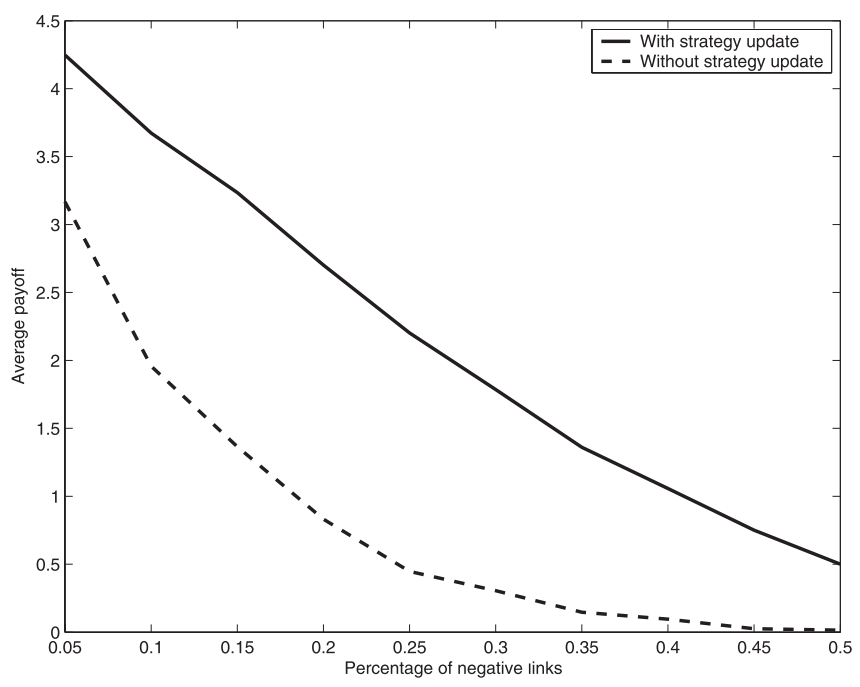

Fig. 11.7. Average payoffs vs. negative links. 
We concentrated only on distributed methods that use local interactions. We developed and analyzed a cooperative game framework first and demonstrated how collaboration can be induced. We showed that negotiation between the mobile agents is an important component for achieving collaboration within this framework. We next developed a model for establishing, propagating and managing trust within a MANET. We showed that such trust mechanisms can also establish collaboration, even without negotiations between the mobile agents. Finally we investigated both the dynamics of games as well as of trust propagation as a means for quantifying the degree of collaboration achieved among the agents and of the speed by which this collaboration spreads in a large part of the network agents. In the context of our research reported here, we have drawn inspiration from analytical methods used in statistical mechanics investigations of the Ising model and spin glasses. these analogies include the existence and investigation of phenomena analogous to phase transitions.

Important current and future directions of our research program are the evaluation of the robustness of these mechanisms for collaboration in wireless networks, analysis of their reliability and identification of parameters (including topology types) that influence the dynamics and the qualities of the induced collaborative behavior.

\section{Acknowledgment}

Prepared through collaborative participation in the Communications and Networks Consortium sponsored by the U.S. Army Research Laboratory under the Collaborative Technology Alliance Program, Cooperative Agreement DAAD19-01-20011. Research also supported by a CIP URI grant from the U.S. Army Research Office under grant No DAAD19-01-1-0494. The U.S. Government is authorized to reproduce and distribute reprints for Government purposes notwithstanding any copyright notation thereon.

\section{References}

[1] J.N. Bearden, The spin glass bead game, Tech. Rept., L.L Thurstone Psychometric Laboratory, The University of North Carolina at Chapel Hill, 2000.

[2] S. Buchegger and J.Y.L. Boudec, The effect of rumor spreading in reputation systems for mobile ad-hoc networks, In: Proceedings of Modeling and Optimization in Mobile, Ad Hoc and Wireless Networks (WiOpt), Sophia-Antipolis, France, 2003.

[3] L. Buttyan and J.P. Hubaux, Stimulating cooperation in self-organizing mobile ad hoc networks, ACM/Kluwer Mobile Networks and Applications, 8:5, 2003.

[4] P. Erdös and A. Rényi, On random graphs I. Publ. Math., 290-297, 1959.

[5] M. Felegyhazi, L. Buttyan and J.P. Hubaux, Equilibrium analysis of packet forwarding strategies in wireless ad hoc networks - the static case. In: Proceedings of Personal Wireless Communications (PWC '03), Venice, Italy, 2003.

[6] F. Forgo, J. Szep and F. Szidarovszky, Introduction to the Theory of Games: Concepts, Methods, Applications. Kluwer Academic Publishers, 1999. 
[7] S.D. Kamvar, M.T. Schlosser and H. Garcia-Molina, The eigentrust algorithm for reputation management in $\mathrm{p} 2 \mathrm{p}$ networks, In: Proceedings of the Twelfth International World Wide Web Conference, 640-651, Budapest, Hungary, 2003.

[8] S. Marti and H. Garcia-Molina, Limited reputation sharing in p2p systems, In: Proceedings of the 5th ACM Conference on Electronic Commerce, 91-101, ACM Press, New York, USA, 2004.

[9] S. Marti, T.J. Giuli, K. Lai and M. Baker, Mitigating routing misbehavior in mobile ad hoc networks, In: Proceedings of the 6th Annual International Conference on Mobile Computing and Networking, 255-265, ACM Press, Boston, MA, USA, 2000.

[10] R.B. Myerson, Game Theory: Analysis of Conflict, Harvard University Press, 1991.

[11] H. Nishimori, Statistical Physics of Spin Glasses and Information Processing: An Introduction, Oxford University Press, 2001.

[12] J. Spencer, The Strange Logic of Random Graphs, Springer, 2001.

[13] V. Srinivasan, P. Nuggehalli, C.F. Chiasserini and R.R. Rao, Cooperation in wireless ad hoc networks, In: Proceedings of IEEE INFOCOM, San Francisco, CA, 2003.

[14] G. Szabo and C. Hauert, Evolutionary prisonner's dilemma games with voluntary participation, Phys. Rev. E, Stat. Nolin. Soft Matter Phys, 66 (6), 2002.

[15] D.J. Watts, A simple model of global cascades on random networks, In: Proceedings of the National Academy of Sciences, 99 (9), 2002.

[16] D.J. Watts, Small Worlds: The Dynamics of Networks Between Order and Randomness, Princeton University Press, 2004. 